\title{
Large copy number variations in combination with point mutations in the TYMP and SCO2 genes found in two patients with mitochondrial disorders
}

\author{
Alžběta Vondráčková ${ }^{1}$, Kateřina Veselá ${ }^{1}$, Hana Kratochvílová ${ }^{1}$, Vendula Kučerová Vidrová ${ }^{1}$, Kamila Vinšová ${ }^{1}$, \\ Viktor Stránecký ${ }^{2}$, Tomáš Honzík ${ }^{1}$, Hana Hansíková ${ }^{1}$, Jiří Zeman ${ }^{1}$ and Markéta Tesařová ${ }^{\star 1}$
}

Mitochondrial disorders are caused by defects in mitochondrial or nuclear DNA. Although the existence of large deletions in mitochondrial DNA (mtDNA) is well known, deletions affecting whole genes are not commonly described in patients with mitochondrial disorders. Based on the results of whole-genome analyses, copy number variations (CNVs) occur frequently in the human genome and may overlap with many genes associated with clinical phenotypes. We report the discovery of two large heterozygous CNVs on 22q13.33 in two patients with mitochondrial disorders. The first patient harboured a novel point mutation c.667G $>$ A (p.D223N) in the SCO2 gene in combination with a paternally inherited 87-kb deletion.

As hypertrophic cardiomyopathy (HCMP) was not documented in the patient, this observation prompted us to compare his clinical features with all 44 reported SCO2 patients in the literature. Surprisingly, the review shows that HCMP was present in only about $50 \%$ of the SCO2 patients with non-neonatal onset. In the second patient, who had mitochondrial neurogastrointestinal encephalopathy (MNGIE), a maternally inherited 175-kb deletion and the paternally inherited point mutation c.261G $>$ T (p.E87D) in the TYMP gene were identified.

European Journal of Human Genetics (2014) 22, 431-434; doi:10.1038/ejhg.2013.148; published online 10 July 2013

Keywords: CNVs; TYMP; SCO2; mitochondrial disorders; cytochrome $c$ oxidase deficiency

\section{INTRODUCTION}

Mutations in mitochondrial DNA (mtDNA) or nuclear genes encoding vital mitochondrial proteins result in a clinically heterogeneous group of mitochondrial disorders, which occur at an incidence of 1 in 5000 neonates. ${ }^{1}$ Importantly, the genetic basis of mitochondrial disorders is still unexplained in many patients, despite encouraging advances in genetic research. ${ }^{2}$

Although the existence of large deletions in mtDNA is well known, deletions affecting the nuclear genes are not commonly described in patients with mitochondrial disorders. A homozygous deletion spanning three genes including NDUFAF2 was found in a patient with fatal multisystem disorder. ${ }^{3}$ Leary et al..$^{4}$ reported heterozygous de novo deletion of the $\mathrm{SCO} 2$ allele in combination with point mutation in a patient with neonatal hypertrophic cardiomyopathy (HCMP). Homozygous or heterozygous deletions of several exons of other OXPHOS-related disease genes were identified by application of targeted array CGH in several patients with mitochondrial diseases. ${ }^{5,6}$

Structural variants of human DNA at least $\geq 1 \mathrm{~kb}$ in length are called copy number variations (CNVs). According to the latest studies, CNVs are significant genetic factors that may influence the apparent phenotype of every individual. ${ }^{7} \mathrm{CNVs}$ can expose sequence variants with an unclear effect in genes of unknown function, change epigenetic factors or alter genomic neighbourhood. ${ }^{8-10}$ These facts together with both the nuclear and mitochondrial genetic backgrounds ${ }^{8,11}$ may further contribute to the complex clinical phenotype of mitochondrial disorders.

In this study, we report the findings of two large heterozygous CNVs on 22q13.33 in combination with TYMP or SCO2 point mutations in two patients with mitochondrial disorders. Moreover, we discuss the phenotype-genotype correlation of our patients considering recent data regarding the heterogeneity of the human genome.

\section{PATIENTS AND METHODS}

Patient 1

The boy was born at term after an uncomplicated delivery as a second child of non-consanguineous parents of Caucasian origin. The birth weight was $3380 \mathrm{~g}$ and the length was $50 \mathrm{~cm}$. The postnatal adaptation was uneventful, and until the age of 7.5 months, he developed normally. Thereafter, myoclonic movements on extremities were noted, and severe hypotonic syndrome developed. Failure to thrive, bilateral ptosis, divergent strabismus and ataxia were observed from the age of 9 months, accompanied by the regression of his psychomotor development. At the age of 14 months, the boy was microcephalic, with a head circumference of $44.5 \mathrm{~cm}$ ( $<3 \mathrm{rd}$ percentile), weight $7.9 \mathrm{~kg}$ ( $<3 \mathrm{rd}$ percentile) and length $79 \mathrm{~cm}$ (50th percentile), and his psychomotor development was on the level of a 6-9-month-old infant. Brain MRI revealed delayed myelination. EMG and echocardiography were normal. Neither hepatopathy nor elevation of creatine kinase was noted. Metabolic investigation revealed mild hyperlactaciduria $(81 \mathrm{mmol} / \mathrm{l}$ creatinine, controls $<30)$ accompanied by increased 
urinary excretion of the Krebs-cycle intermediates (fumarate $21 \mathrm{mg} / \mathrm{g}$ creatinine, controls $<15$; aconitate $150 \mathrm{mg} / \mathrm{g}$ creatinine, controls $<100$; 2-oxoglutarate $216 \mathrm{mg} / \mathrm{g}$ creatinine, controls $<200$ ). Lactate concentration was elevated in CSF $(3 \mathrm{mmol} / \mathrm{l}$, controls $<2)$. The patient died due to further deterioration of the disease at the age of 20 months.

\section{Patient 2}

The boy was born at term after an uncomplicated delivery as a first child of non-consanguineous parents of Caucasian origin. The birth weight was $3550 \mathrm{~g}$ and the length was $52 \mathrm{~cm}$. The postnatal adaptation was uneventful. Severe failure to thrive developed at the age of 4-5 years. At the age of 10 years, his weight was only $16 \mathrm{~kg}$. He had frequent attacks of abdominal pain and alternation of diarrhoea and constipation due to gastrointestinal dysmotility, leading to extreme nausea and intermittent vomiting. X-ray investigation of the gastrointestinal tract found jejunal diverticulosis. Physical endurance was diminished: he was not able to climb stairs and his ability to ride a bicycle was very limited. At the age of 12 years, brain MRI showed white matter abnormalities compatible with the diagnosis of leukodystrophy, and an EMG study revealed polyneuropathy. At the age of 13 years, his weight was $17.5 \mathrm{~kg}$ $(-4.67 \mathrm{SD})$, height $135 \mathrm{~cm}(-3.78 \mathrm{SD})$ and head circumference $52 \mathrm{~cm}$ (3rd percentile). Neurological investigation documented severe hypotonic syndrome, general hyporeflexia and muscle hypotrophy. Psychological investigation showed normal mental capabilities. Retinitis pigmentosa developed at the age of 16 years. At present, the 16-year-old boy is on total parenteral nutrition, and his weight is $24 \mathrm{~kg}$. The severe gastrointestinal dysmotility precludes any enteral feeding. He is wheelchair-bound. He attends school on an intermittent basis. There was neither hepatopathy nor elevation of creatine kinase. During acute gastroenteritis, protein-losing enteropathy developed, leading to hypoalbuminemia ( $19 \mathrm{~g} / \mathrm{l}$, controls $35-53)$ with oedema and ascites. Metabolic investigation revealed mild hyperlactaciduria $(81 \mathrm{mmol} / \mathrm{l}$ creatinine, controls $<30$ ) accompanied by increased urinary excretion of the Krebs-cycle intermediates (fumarate $18 \mathrm{mg} / \mathrm{g}$ creatinine, controls $<15$; aconitate $140 \mathrm{mg} / \mathrm{g}$ creatinine, controls $<100)$ and 3-methylglutaconic aciduria $(45 \mathrm{mg} / \mathrm{g}$ creatinine, controls <15). The lactate concentration in blood was not elevated. We found increased levels of uracil $(93 \mathrm{mmol} / \mathrm{mol}$ creatinine, controls $<15)$, thymine $(15.4 \mathrm{mmol} / \mathrm{mol}$ creatinine, controls $<15)$, deoxyuridine $(39 \mathrm{mmol} /$ mol creatinine, controls not detectable) and thymidine $(12.6 \mathrm{mmol} / \mathrm{mol}$ creatinine, controls not detectable).

\section{Molecular genetic analysis}

Genomic DNA was extracted from peripheral blood lymphocytes using standard procedures. The nuclear DNA of these patients was analysed using a Genome-Wide Human SNP 6.0 microarray chip (Affymetrix, Santa Clara, CA, USA). The presence of just one copy of the SCO2 and TYMP genes in both affected patients was verified by a real-time PCR CNV assay (Hs00093549_cn, Hs00001601_cn, Hs00137275_cn, Hs00574610_cn; Applied Biosystems, Foster City, CA, USA). Sequencing of coding exons and their flanking intronic regions of SCO2 (NG_016235.1) and TYMP (NG_011860.1) PCR products was performed in both directions on an ABI PRISM 3100/3100-Avant Genetic Analyser (Applied Biosystems). The identified missense mutations were verified by PCR-RFLP analysis, and their impact was evaluated by in silico analysis methods using the PolyPhen-2 (http://genetics.bwh.harvard.edu/ pph2), MutPred (http://mutpred.mutdb.org) and SNPs\&GO (http://snpsand-go.biocomp.unibo.it/snps-and-go) databases.

Other methods used are described in Supplementary file 1 Methods.

\section{Ethics}

This study was approved by the Ethics Committee of the General University Hospital in Prague. All blood and tissue samples were analysed with the informed consent of the parents of the patients.

\section{RESULTS}

In patient 1 , a paternally inherited $87-\mathrm{kb}$ deletion on Chr. 22: g. [(49275958_49362964)del (NCBI Build 36.1)] spanning eight genes (LMF2, NCAPH2, SCO2, TYMP, ODF3B, KLHDC7B, c22orf41 and
$C P T 1 B$ ) was identified by microarrays (see Supplementary Figure 1) and confirmed by a real-time PCR CNV assay (see Supplementary Figure 2). This deletion corresponds to a known CNV-variation_4139, which occurs with a frequency of $\sim 0.4 \%$ in control samples. ${ }^{12}$ In combination with this deletion, a novel point mutation c.667G > A (p.Asp223Asn) in the SCO2 gene was found. The alignment of SCO2 proteins from multiple species showed that the affected Asp223 is completely conserved. The novel missense SCO2 mutation was not present in 100 Czech healthy control samples. No pathogenic mutation was found in the TYMP gene. In muscle and heart mitochondria, a markedly decreased amount of SCO2 protein was found by immunoblot analysis (Figure 1). Lower levels of copper content (Table 1) were detected in autoptic liver ( $10 \%$ of control value), brain, heart and muscle (60-70\% of control value), further suggesting cytochrome $c$ oxidase (COX) deficiency due to SCO2 dysfunction ${ }^{13}$ in this patient. In addition, the pathogenicity of this mutation was predicted by in silico analysis with PolyPhen-2, MutPred and SNPs\&GO.

In patient 2, a maternally inherited $175-\mathrm{kb}$ deletion on Chr. 22: g.[(49275958_49451008)del (NCBI Build 36.1)] spanning 12 genes (LMF2, NCAPH2, SCO2, TYMP, ODF3B, KLHDC7B, c22orf41, CPT1B, CHKB, LOC100144603, MAPKIP2 and ARSA) along with the paternally inherited point mutation c.261G $>$ T (p.Glu87Asp) in the TYMP gene were identified (see Supplementary Figure 1). Although the nucleotide substitution c.261G $>\mathrm{T}$ has not been described previously, the resulting amino-acid replacement p.Glu87Asp has already been characterised. ${ }^{14}$ The $175-\mathrm{kb}$ deletion spans 12 genes and corresponds to known CNV-variation_5192, which occurs with almost $7 \%$ frequency in control samples. ${ }^{12}$ In this
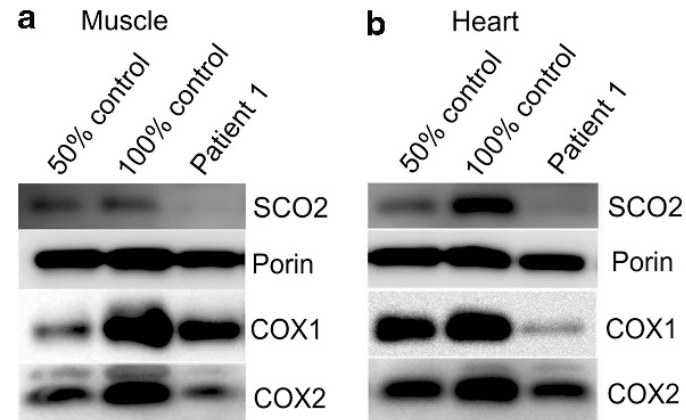

Figure 1 Steady-state levels of SCO2 protein in muscle (a) and heart (b) mitochondria of patient 1. A total of $10 \mu \mathrm{g}$ of mitochondrial protein from muscle and heart mitochondria was separated using SDS-PAGE (12\% polyacrylamide), electroblotted onto PVDF membranes and detected with polyclonal antiserum raised against human $\mathrm{SCO} 2$ or with monoclonal antibody raised against the COX1, COX2 subunits and porin. Two aliquots of control mitochondria corresponding to indicated dilutions of control samples were loaded on the same gels.

Table 1 Total copper content in autoptic tissues of patient 1 with SCO2 deficiency (expressed in $\mu \mathrm{g}$ in $1 \mathrm{~g}$ of dry tissue)

\begin{tabular}{lcc}
\hline & & Copper content $(\mu \mathrm{g} / \mathrm{g})$ \\
& Patient 1 & Controls \\
\hline Muscle & 1.12 & $1.74 \pm 0.53(n=9)$ \\
Heart & 2.2 & $2.98 \pm 0.87(n=8)$ \\
Brain (frontal cortex) & 2.26 & $3.06 \pm 1.62(n=6)$ \\
Liver & 4.49 & $49.03 \pm 24.01(n=10)$
\end{tabular}


Table 2 Biochemical markers assessed in patient 2 with MNGIE

\begin{tabular}{lcc}
\hline Biochemical markers & Patient 2 & Controls \\
\hline Thymidine in plasma $(\mu \mathrm{mol} / \mathrm{l})$ & 16.5 & $<0.05$ \\
Thymidine in urine $(\mathrm{mmol} / \mathrm{mol}$ of creatinine) & 12.6 & Not detected \\
TP activity in lymphocytes $(\mu \mathrm{mol} / \mathrm{mg} / \mathrm{h})$ & 0.002 & $0.41-0.95(n=14)$ \\
Cytochrome $c$ oxidase $(\mathrm{COX})$ activity in lymphocytes $(\mathrm{nmol} / \mathrm{mg}$ of protein per $1 \mathrm{~min})$ & 19 & $21-43(n=51)$ \\
Citrate synthase $(\mathrm{CS})$ activity in lymphocytes $(\mathrm{nmol} / \mathrm{mg}$ of protein per $1 \mathrm{~min})$ & 75 & $49-93$ \\
COX/CS & 0.24 & $0.31-0.65$ \\
\hline
\end{tabular}

Abbreviation: MNGIE, mitochondrial neurogastrointestinal encephalopathy.

patient, the mitochondrial neurogastrointestinal encephalopathy (MNGIE) diagnosis was biochemically confirmed by the diminished thymidine phosphorylase activity in patient lymphocytes. The levels of thymidine in plasma and urine were increased, and the activity of COX and its ratio to citrate synthase (CS) activity in lymphocytes were decreased (Table 2). No pathogenic mutation was found in the SCO2 gene.

To evaluate the occurrence of CNVs spanning the $\mathrm{SCO} 2$ gene in the Czech population, a real-time PCR CNV assay was carried out in 50 control samples. However, no CNV encompassing SCO2 was found in the 50 tested Czech control samples (see Supplementary Figure 3).

\section{DISCUSSION}

We described two large CNVs on chromosome 22q13.33 that span up to 12 genes found in two patients with mitochondrial disorders. According to the Database of Genomic Variants, only three documented CNVs of $\sim 87-175 \mathrm{~kb}$ on chromosome 22q13.33 and overlapping the TYMP and SCO2 genes, including CNV-variation_4139 and CNV-variation_5192 found in the presented patients, have been identified so far with total frequency of $2.2 \%$ in control samples. ${ }^{12}$ Only 2 out of the 12 genes, apart from SCO2 and TYMP, affected by the deletions are associated with human disease (Supplementary Table 1). Although mutations in both ARSA and CHKB result in autosomal recessive disorders, their clinical features, neonatal manifestation of cardiomyopathy with increased creatine kinase activity found in $C H K B$-related muscular dystrophy ${ }^{15}$ or mental decline specific for juvenile form of metachromatic leukodystrophy, are distinct from symptoms observed in our patients.

In patient 1 , we diagnosed $\mathrm{SCO} 2$ deficiency based on genetic analyses. With regard to knowledge of the human $\mathrm{SCO} 2$ protein structure, the newly identified hemizygous mutation p.D223N is likely to affect the coordination of copper (I) by the two cysteines of the CXXXC conserved motif and by the conserved His $224 .{ }^{16}$ Furthermore, markedly decreased levels of SCO2 protein and border-low copper content found in tissues of patient 1 are in accordance with previously reported findings in SCO2 patients. ${ }^{13,17}$ To date, we have diagnosed the late non-neonatal onset of SCO2 deficiency in eight patients, ${ }^{18}$ but ataxia was not noted in any of these or other reported SCO2 patients (Supplementary Table 2). All of the observed symptoms are typical of $\mathrm{SCO} 2$ deficiency. Interestingly, based on the review of published cases, HCMP, which is the key finding in SCO2-deficient patients with neonatal onset, is present only in about $50 \%$ of the SCO2 patients with non-neonatal presentation (Supplementary Table 2).

With regard to the biochemical and genetic findings, MNGIE was diagnosed in patient 2 . The manifested symptoms of patient 2 were similar to those described previously. ${ }^{19}$ Whereas no pathogenic mutation was found in the SCO2 gene of patient 2, we assume that mildly decreased COX activity in his lymphocytes might be a consequence of point mtDNA-mutation accumulation due to diminished thymidine phosphorylase activity as reported previously in MNGIE patients. ${ }^{20}$

The number of mitochondrial proteins in mammals has been estimated to be between 1130 and 1500, but pathogenic defects have been found in only a fraction of these proteins. ${ }^{2}$ Additionally, there is no satisfactory explanation for the phenotype-genotype variability in patients suffering from mitochondrial diseases. However, we believe that the use of advanced whole-genome analysis techniques will shed light on the genetic causes of mitochondrial defects, as well as on the regulation of tissue-specific energy demands. ${ }^{9,10}$ Further improvement in our knowledge of mechanisms based on the presence of variable gene dosage, epigenetic modifications, repetitive sequences, noncoding RNAs, cis- and trans-splicing, diverse penetrance of diseases, the complex effects of the hundreds of loss-of-function variants present in every individual, and general agreement on the classification of particular genetic variants will clarify the phenotypic diversity in humans. $^{21,22}$

\section{CONFLICT OF INTEREST}

The authors declare no conflict of interest.

\section{ACKNOWLEDGEMENTS}

We thank the patients and their families for participating in the study. We thank T Adam and V Smolka for referring patient 2 to our clinic. This study was supported by the grants GAUK 28410 and SVV266504 from the Charles University in Prague; IGA NT13114/4 from the Internal Grant Agency of the Ministry of Health of the Czech Republic; and by the research projects P24/LF1/3 and UNCE 204011 from the Charles University in Prague.

1 Schafer AM, Taylor RW, Turnbull DM, Chinnery PF: The epidemiology of mitochondrial disorders - past, present, future. Biochim Biophys Acta 2004; 1659 : $115-120$.

2 Koopman WJ, Willems PH, Smeitink JA: Monogenic mitochondrial disorders. N Engl J Med 2012; 366: 1132-1141.

3 Janssen RJ, Distelmaier F, Smeets R et al: Contiguous gene deletion of ELOVL7, ERCC8 and NDUFAF2 in a patient with a fatal multisystem disorder. Hum Mol Genet 2009; 18: 3365-3374.

4 Leary SC, Mattman A, Wai T et al: A hemizygous SCO2 mutation in an early onset rapidly progressive, fatal cardiomyopathy. Mol Genet Metab 2006; 89: 129-133.

5 Compton AG, Troedson C, Wilson M et al: Application of oligonucleotide array CGH in the detection of a large intragenic deletion in POLG associated with Alpers Syndrome. Mitochondrion 2011; 11: 104-107.

6 Wang J, Zhan H, Li FY, Pursley AN, Schmitt ES, Wong LJ: Targeted array CGH as a valuable molecular diagnostic approach: experience in the diagnosis of mitochondrial and metabolic disorders. Mol Genet Metab 2012; 106: 221-230.

7 Scherer SW, Lee C, Birney E et al: Challenges and standards in integrating surveys of structural variation. Nat Genet 2007; 39: S7-S15.

8 De S: Somatic mosaicism in healthy human tissues. Trends Genet 2011; 27: 217-223.

9 Chinnery PF, Elliott HR, Hudson G, Samuels DC, Relton CL: Epigenetics, epidemiology and mitochondrial DNA diseases. Int J Epidemiol 2012; 41: 177-187.

10 De S, Babu MM: Genomic neighbourhood and the regulation of gene expression. Curr Opin Cell Biol 2010; 22: 326-333. 
11 Pello R, Martin MA, Carelli V et al: Mitochondrial DNA background modulates the assembly kinetics of OXPHOS complexes in a cellular model of mitochondrial disease. Hum Mol Genet 2008; 17: 4001-4011.

12 lafrate AJ, Feuk L, Rivera MN et al: Detection of large-scale variation in the human genome. Nat Genet 2004; 36: 949-951.

13 Stiburek L, Vesela K, Hansikova H, Hulkova H, Zeman J: Loss of function of Scol and its interaction with cytochrome c oxidase. Am J Physiol Cell Physiol 2009; 296: C1218-C1226.

14 Slama A, Lacroix C, Plante-Bordeneuve $\mathrm{V}$ et al: Thymidine phosphorylase gene mutations in patients with mitochondrial neurogastrointestinal encephalomyopathy syndrome. Mol Genet Metab 2005; 84: 326-331.

15 Nishino I, Kobayashi O, Goto $Y$ et al: A new congenital muscular dystrophy with mitochondrial structural abnormalities. Muscle Nerve 1998; 21: 40-47.

16 Banci L, Bertini I, Ciofi-Baffoni S et al: A structural characterization of human SCO2. Structure 2007; 15: 1132-1140.
17 Stiburek L, Vesela K, Hansikova $\mathrm{H}$ et al: Tissue-specific cytochrome c oxidase assembly defects due to mutations in SCO2 and SURF1. Biochem J 2005; 392: 625-632.

18 Vesela K, Hansikova H, Magner M, Zeman J: Cytochrome c oxidase deficiency in childhood. Paediatr Croat 2009; 53: 122-126.

19 Garone C, Tadesse S, Hirano M: Clinical and genetic spectrum of mitochondrial neurogastrointestinal encephalomyopathy. Brain 2011; 134: 3326-3332.

20 Nishigaki Y, Marti R, Copeland WC, Hirano M: Site-specific somatic mitochondrial DNA point mutations in patients with thymidine phosphorylase deficiency. J Clin Invest 2003; 111: 1913-1921.

21 Riggs ER, Church DM, Hanson $\mathrm{K}$ et al: Towards an evidence-based process for the clinical interpretation of copy number variation. Clin Genet 2012; 81: 403-412.

22 Altshuler DM, Gibbs RA, Peltonen L et al: Integrating common and rare genetic variation in diverse human populations. Nature 2010; 467: 52-58.

Supplementary Information accompanies this paper on European Journal of Human Genetics website (http://www.nature.com/ejhg) 